\title{
Diferencias de género epidemiológicas y diagnósticas según eje I en pacientes con Patología Dual
}

\section{Epidemiological and diagnostic axis I gender differences in Dual Diagnosis patients}

\author{
| Laia Miquel ${ }^{*},{ }^{* *}$; CARlos Roncero*, ${ }^{* *},{ }^{* \star *}$; CRISTINA \\ lóPeZ-OrTiZ ${ }^{\star *}$; Miguel CASAS ${ }^{\star *},{ }^{* *}$
}

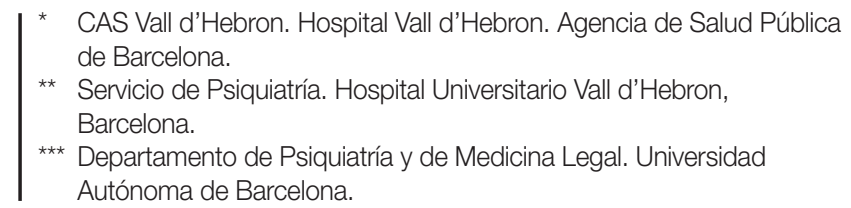

Enviar correspondencia a:

Laia Miquel de Montagut. CAS Vall d'Hebron; Hospital Universitario

Vall d'Hebron. Servicio de Psiquiatría.

Passeig Vall d'Hebron, 119-129; 08035; Barcelona.

TEL: 934893 880; FAX: 934894587.

Correo electrónico: lamiquel@vhebron.net

\section{RESUMEN}

Introducción: La patología dual (PD) es la coexistencia de un trastorno por uso de sustancias (TUS) y otro trastorno mental. Se ha descrito que el género es un factor que influye en la prevalencia de las enfermedades psiquiátricas, su expresión sintomática, curso, pronóstico, demanda y respuesta al tratamiento.

Material y métodos: Se revisan 40 estudios publicados en Medline, Web of Science, y Journal Citation Reports hasta diciembre de 2009 que contienen información a cerca de las diferencias de género en la prevalencia y las caracteristicas clínicas de pacientes duales mayores de 18 años.

Conclusiones: La distribución de los trastornos mentales del Eje I en pacientes duales, según el género, se mantiene similar a la de los trastornos mentales no duales en la población general. Existe mayor prevalencia en hombres de trastornos psicóticos y bipolares, y en mujeres mayor prevalencia de trastornos de ansiedad y afectivos. El género femenino deja de ser factor de buen pronóstico en la psicosis dual. Por otra parte, el policonsumo es más frecuente en los varones.

Palabras Clave: género, patología dual, mujer, adicción, trastorno por uso de sustancias.

\section{ABSTRACT}

Introduction: Dual diagnosis is the co-occurrence of a substance abuse disorder and a psychiatric condition. Gender has been found to be associated with differences in prevalence of mental disorders as well as outcome, prognosis and treatment-seeking. Material and Methods: Articles published in Medline, Web of Science and Journal Citation Reports up to December 2009 that examined gender, prevalence and clinical characteristics of dual-diagnosis patients aged over 18 were reviewed.

Conclusions: The distribution of Axis I disorders by gender is similar for dually diagnosed patients and single-disorder patients. The prevalence of psychotic and bipolar disorders is higher in men, whereas anxiety and affective disorders are more prevalent in women. Dually diagnosed females with psychotic disorders do not show better prognosis than men. Finally, polydrug use among dual-diagnosis individuals is more prevalent in males.

Keywords: gender, dual diagnosis, women, addiction, substance use disorder. 


\section{INTRODUCCIÓN}

L a patología dual (PD) es la coexistencia de un trastorno mental y un trastorno por uso de sustancias (TUS). La etiopatogenia sigue siendo desconocida, aunque se cree que están implicados diferentes factores biopsicosociales. Los estudios epidemiológicos han podido objetivar que la prevalencia de la patología dual es elevada (Regier, Farmer, Rae, Locke, Keith, Judd et al., 1990). El 21\% de pacientes psicóticos atendidos en urgencias, el $37 \%$ de pacientes con primeros episodios psicóticos, y el $24 \%$ de pacientes ingresados en unidades psiquiátricas presentó uno o más diagnósticos de uso de alcohol y drogas ilegales (Cantwell, Brewin, Glazebrook, Dalkin, Fox, Medley et al., 1999; Claassen, Gilfillan, Orsulak, Carmody, Battaglia, y Rush, 1997; Rodríguez-Jimenez, Aragues, Jimenez-Arriero, Ponce, Muñoz et al., 2008).

Los estudios realizados en pacientes con patología dual observaron que los trastornos psicóticos fueron el diagnóstico más frecuente $(53,1 \%$ trastorno esquizofreniforme, $28,1 \%$ esquizofrenia) (RodríguezJimenez et al., 2008). Sin embargo, en pacientes diagnosticados de TUS los trastornos afectivos fueron la patología psiquiátrica más prevalente (DiNitto, Webb, y Rubin, 2002; Sanabria, Díaz, Varela, y Brana, 2002). Aunque en este punto no todos los trabajos coinciden, siendo los trastornos de ansiedad los más frecuentes $(65 \%)$ seguido de los trastornos afectivos (33\%) y el trastorno de personalidad antisocial (28\%) (Tomasson y Vaglum, 1995). En España, los trastornos afectivos fueron los más frecuentes en pacientes drogodependientes $(21,6 \%)$ seguido de los trastornos de ansiedad $(11,7 \%)$ y la esquizofrenia $(3,1 \%)$ (Gual, 2007).

Se ha descrito que la condición de hombre o mujer influye en la prevalencia de las enfermedades psiquiátricas, la patoplastia, la expresión de los síntomas, el curso, el pronóstico, la demanda y la respuesta al tratamiento (Gearon, Nidecker, Bellack, y Bennette, 2003b; Seedat, Scott, Angermeyer, Berglund, Bromet, Brugha et al., 2009). En las últimas décadas, el interés acerca de la influencia del género en las enfermedades mentales y los TUS se ha ido incrementando. La mayoría de estudios han evaluado las diferencias de sexo en muestras de pacientes con patología mental y TUS por separado, siendo escasos los trabajos en pacientes con patología dual. Hasta la actualidad existen datos discrepantes debido a la variabilidad de los resultados que podría estar relacionada con la heterogeneidad de las poblaciones estudiadas, los instrumentos diagnósticos y de evaluación del consumo, y el estado psicopatológico. Aunque se cree que la patología dual es más frecuente en hombres, se considera que las mujeres tienen más riesgo de presentar ambas patologías.

El objetivo del presente trabajo es revisar las diferencias de género previamente descritas a nivel epidemiológico, psicopatológico y diagnóstico en pacientes duales que presentan un trastorno del eje I.

\section{METODOLOGÍA}

Se realizó una revisión de artículos publicados en la base de datos MEDLINE, Web of Science, y Journal Citation Reports hasta diciembre de 2009. Se utilizó la combinación de los siguientes términos de búsqueda ("dual diagnosis", "gender", "gender differences", "substance", "mental disorders", "mental illness", "psychosis", "depression", "bipolar disorder", "obsessive-compulsive disorder", "post-traumatic stress disorder", "anxiety", "generalized anxiety disorder"). Se limitó a los artículos en español, inglés y francés, en población mayor de 18 años. Por otro lado, se revisaron las referencias bibliográficas citadas en las revistas seleccionadas. Se incluyeron aquellos artículos que estudiaron muestras poblacionales de pacientes ingresados o en tratamiento ambulatorio de las redes de salud mental y adicciones con diagnóstico dual. Se revisaron los artículos que analizaron las diferencias de género en variables sociodemográficas (raza, estado civil, nivel académico, actividad laboral, funcionamiento social) y clínicas (prevalencia psiquiátrica, edad de inicio de la enfermedad del eje I, tipo de sustancia consumida, número de sustancias consumidas, tipo de diagnóstico, y gravedad clínica). Se excluyeron los artículos cuya muestra estaba formada exclusivamente por pacientes con trastorno de personalidad comórbido al TUS y aquellos en los que no se especificaba el porcentaje de pacientes que presentaba patología dual. También se excluyeron aquellos artículos que sólo realizaron comparaciones entre pacientes duales y no duales sin considerar el género. No se analizaron los estudios que tenían como variables de resultado adherencia al tratamiento, uso de recursos asistenciales, nivel cognitivo, o los que no usaron criterios DSM, ni CIE. De los 218 artículos 40 cumplieron los criterios de selección.

\section{DIFERENCIAS DE GÉNERO}

Los estudios realizados en pacientes con patología dual observaron que la esquizofrenia es más frecuente entre los hombres (57,7\% vs 38\%, p<0,01) (Comtois y Ries, 1995; Westreich, Guedj, Galanter, y Baird, 1997) y los trastornos afectivos en las mujeres, principalmente el trastorno depresivo mayor (TDM) $(16,55 \%$ vs 10,2\%; $p<0,01)$ (Comtois y Ries, 1995).

En las unidades de psiquiatría general hay más hombres duales que mujeres $(87,5 \%$ varones vs. $12,5 \%$ mujeres, $p<0,001$ ) (Rodriguez-Jimenez et al., 2008). Sin embargo, cuando se estudian muestras de pacientes en unidades de desintoxicación o en seguimiento ambulatorio de adicciones se invierte la relación observándose mayor porcentaje de patología dual en las mujeres que en los hombres (Tabla 1).

En España, un estudio realizado mayoritariamente en población atendida en unidades de drogodependencias observó que el 47,5\% de las mujeres presentaba patología dual, mientras que sólo el 30,3\% de los hombres; $p=0,001$ (Gual, 2007). Aunque en números absolutos hay más hombres duales que mujeres los datos sugieren, coincidiendo con otros autores, que las mujeres consumidoras tuvieron 
Tabla 1. Diferencias de género en la prevalencia de la patología dual

\begin{tabular}{|c|c|c|c|c|c|}
\hline Autor & Año & Muestra & Instrumentos & Droga & Resultados \\
\hline Brady et al. & 1993 & $\begin{array}{l}100(50 \mathrm{H}, 50 \mathrm{M}) \\
\text { ingresados U. DTX }\end{array}$ & SCID & $\begin{array}{l}\text { Cocaina } \\
\text { Alcohol }\end{array}$ & $70 \%$ mujeres vs. $48 \%$ hombres; $p<0,005$. \\
\hline Chiang et al. & 2007 & $\begin{array}{c}155(123 \mathrm{H}, 32 \mathrm{M}) \\
\text { Tratamiento ambulatorio }\end{array}$ & MINI & Heroína & $62,5 \%$ mujeres vs. $58,5 \%$ hombres; $p<0,05$. \\
\hline Landheim et al. & 2003 & $\begin{array}{l}260 \text { ingresados y en tratami- } \\
\text { ento ambulatorio con TUS }\end{array}$ & $\begin{array}{c}\text { CIDI } \\
\text { MCMI-II }\end{array}$ & $\begin{array}{l}\text { Drogas } \\
\text { ilegales y } \\
\text { legales }\end{array}$ & $96 \%$ mujeres vs. $82 \%$ hombres; $p<0,01$. \\
\hline $\begin{array}{l}\text { Tomasson y } \\
\text { Vaglum }\end{array}$ & 1995 & $\begin{array}{c}351 \text { Pacientes ingresados } \\
\text { U. DTX. } \\
(71 \% \mathrm{H})\end{array}$ & $\begin{array}{c}\text { DIS } \\
\text { MMSE } \\
\text { DSM-III-R }\end{array}$ & $\begin{array}{c}\text { Alcohol } \\
\text { otras drogas. }\end{array}$ & $82 \%$ mujeres vs. $74 \%$ hombres, $p<0,0001$. \\
\hline Zlotnick et al. & 2008 & $\begin{array}{c}272 \text { pacientes en prisión } \\
\text { con TUS } \\
(66 \% \mathrm{H})\end{array}$ & $\begin{array}{l}\text { CJDATS } \\
\text { SCID }\end{array}$ & $\begin{array}{l}\text { Drogas } \\
\text { ilegales y } \\
\text { legales }\end{array}$ & $\begin{array}{l}64,1 \% \text { mujeres vs. } 35 \% \text { hombres; } p<0,0001 \text {, ajustado } \\
\text { para variables sociodemográficas. } \\
\text { A lo largo de la vida el } 46,7 \% \text { de las mujeres } \\
\text { presentaron uno o más trastornos comparado con los } \\
\text { hombres } 24,7 \%(\mathrm{OR} 2,7) \text {. }\end{array}$ \\
\hline
\end{tabular}

H:hombres; M:mujeres: U. DTX: Unidad de desintoxicación.

más psicopatología que los hombres (Brady, Grice, Dustan, y Randall, 1993; Denier, Thevos, Latham, y Randall, 1991). Al mismo tiempo, las mujeres con un trastorno mental (TM) presentaron una odds de 1,9-2,4 de tener un TUS respecto a las que no lo tenían $(p<0,01)$ (Abram, Teplin, y McClelland, 2003). Concluyendo que las mujeres presentan mayor riesgo de tener ambos trastornos al mismo tiempo (Chiang, Chan, Chang, Sun, Chen, y Chen, 2007).

Las diferencias de género a nivel sociodemográfico en pacientes con patología dual son escasas. No se encontraron diferencias significativas en edad, raza, lugar de residencia en aquellos pacientes con diagnóstico de esquizofrenia, trastorno esquizoafectivo, trastorno bipolar ni trastorno depresivo mayor dual (Comtois y Ries, 1995; Westreich et al., 1997). Tampoco se encontraron diferencias en el nivel de estudios de los pacientes duales con esquizofrenia y trastorno esquizoafectivo (Brunette y Drake, 1997; Gearon y Bellack, 2000; Gearon et al., 2003b).

A nivel laboral los resultados parecen contradictorios. Un estudio no encontró diferencias significativas a excepción de que las mujeres recibieron de forma significativa más pensiones públicas que los hombres (61\% vs 17\%) (DiNitto et al., 2002). Mientras que, por otro lado, se observó que significativamente más hombres que mujeres trabajaban a tiempo completo o parcial $\left(67,7 \%\right.$ vs. $44,0 \%, \chi^{2}=18,89$; $p<0,001)$. Refiriendo los hombres, mayor número de problemas en el trabajo en el último mes (media de días $=13,23$ vs. 8,33; $F=13,02 ; p<0,001$ ) (Mowbray, Ribisl, Solomon, Luke, y Kewson, 1997).

Las mujeres, al igual que en estudios en pacientes con TUS o patología psiquiátrica no dual, tenían más hijos de media (2,13 vs. 1,$46 ; F=10,19 ; p<0,002)$ y éstos estaban a su cargo (media $=0,71$ vs. 0,$30 ; F=18,15 ; p<0,001$ ) (Mowbray et al., 1997). El $72 \%$ de las mujeres vs el $41 \%$ de los hombres tenían domicilio propio $(p<0,05)$ (Westreich et al., 1997).

El porcentaje de mujeres $(40 \%)$ que vivía con una pareja consumidora era mayor que el de hombres (20\%), (phi = 0,25); lo que puede ser un factor de mantenimiento del consumo (29\% frente al $0 \%$ de los hombres; $p<0,01$ ) (DiNitto et al., 2002). Los hombres presentaron más problemas legales que las mujeres (DiNitto et al., 2002), siendo la proporción de arrestos mayor en éstos $(68,2 \%$ vs. $\left.44,47 \% ; \chi^{2}=22,06 ; p<0,001\right)$ al igual que la gravedad de los problemas legales ( $M=3,53$ vs. 2,$69 ; F=9,73 ; p<0,002)$ (Mowbray et al., 1997). El $32 \%$ de las mujeres y el $12 \%$ de los hombres fueron víctimas de crímenes $(p<0,05)$ (Westreich et al., 1997).

Las diferencias entre el consumo de cannabis en población dual difiere según los estudios, así como el consumo de opiáceos. Algunos trabajos detectaron únicamente una ligera tendencia, en los hombres, de mayor consumo de marihuana $\left(\chi^{2}=3,46 ; p<0,10\right)$ (Comtois $y$ Ries, 1995; Mueser, Yarnold, y Bellack, 1992)), mientras que otros observaron significativamente mayor dependencia de marihuana en los hombres ( $54 \%$ vs $18 \% ; p<0,02$ ) (Gearon et al., 2003b). Por otro lado, en las mujeres el consumo de heroína fue mayor (22\% vs 0\%; $<<0,02)$ (Gearon et al., 2003b) en contraposición a otros estudios donde el consumo de opiáceos fue mayor en los hombres (Mueser et al., 1992). El consumo de alcohol en los pacientes varones duales fue mayor y el de estimulantes sólo fue superior en las mujeres diagnosticadas de trastorno esquizoafectivo, sin encontrarse diferencias significativas para el abuso de sedantes (Mueser 
et al., 1992), ni tampoco para el consumo de cocaína (Westreich et al., 1997). Tampoco se encontraron diferencias en las razones por las cuales consumen hombres y mujeres (Gearon et al., 2003b) ni en la gravedad de la adicción evaluado por el Addiction Severity Index (ASI) (Gearon et al., 2003b).

Varios estudios coinciden en que de forma significativa los hombres presentan más policonsumo que las mujeres $(66,5 \%$ hombres vs $47,1 \%$ mujeres, $p<0,002)$ (Comtois y Ries, 1995); $13 \%$ de los hombres y el $9 \%$ de las mujeres (Gearon et al., 2003b).

Queda por dilucidar si las mujeres debutan en la patología dual a edades más tempranas. Algunos estudios realizados en pacientes duales no observaron diferencias de género significativas en la edad de inicio del consumo de drogas (Brunette y Drake, 1997; Gearon et al., 2003b). Un estudio realizado con una muestra de 467 pacientes duales ingresados (28\% esquizofrenia y trastorno esquizoafectivo, 14\% trastorno depresivo mayor, 15\% trastorno afectivo menor, 26\% trastornos adaptativos o trastornos de personalidad, 21\% trastornos afectivos orgánicos) observó que la mayor proporción de mujeres (62,9\% vs. 48,8\%, $\chi^{2}$ $=8,30, p<0,004)$ estaba en el grupo de menos de 34 años (Mowbray et al., 1997).

El funcionamiento social de los hombres duales fue peor que el de las mujeres $(t=-2,84 ; p<0,01)$, posiblemente debido a que entre los hombres es más frecuente el diagnóstico de esquizofrenia (Comtois y Ries, 1995).

El riesgo de presentar infecciones de transmisión sexual, como el VIH, es 3,8 veces mayor en la mujer dual que en el hombre (Krakow, Galanter, Dermatis, y Westreich, 1998). Posiblemente debido a que para conseguir drogas las mujeres recurren con frecuencia a la venta de sexo (26\% vs 0\% p $<0,02$ (Gearon et al., 2003b). Los resultados del ASI para problemas médicos fue contradictorio, algunos autores no encontraron diferencias de género (DiNitto et al., 2002), mientras que otros observaron más problemas en las mujeres $(M=3,55$ vs. 2,$84 ; F=7.87 ; p<0,005)$ (Mowbray et al., 1997).

\section{Psicosis}

La Psicosis dual es muy frecuente. El $47 \%$ de los pacientes diagnosticados de esquizofrenia o trastorno esquizofreniforme presentó a lo largo de la vida un diagnóstico de TUS, siendo el alcohol el más consumido. La Odds de los esquizofrénicos es 4,6 veces superior a los de la población general de tener un TUS (Regier et al., 1990).

En una muestra de 67 pacientes diagnosticados de esquizofrenia y trastorno esquizoafectivo, de los cuales 34 eran duales, no se observaron diferencias de género en la edad de inicio de la patología psicótica dual entre los dos sexos, siendo el tamaño del efecto pequeño $(d=0,2)$ (Gearon y Bellack, 2000). Aunque en ambos sexos la edad de inicio fue más precoz en los pacientes psicóticos duales que en los no duales $(F(1,66)=5,17, p<0,02)$.
El riesgo de consumir alcohol y cannabis es mayor en los varones diagnosticados de esquizofrenia que en las mujeres $(F=13,9 ; R=0,18 ; p<0,05)$ (Mueser, Yarnold, Levinson, Singh, Bellack, Kee et al., 1990). El 26,2\% de los hombres presentaron dependencia a cannabis frente al $8 \%$ de las mujeres $(p=0,008)$ (Brunette y Drake, 1997). El impacto del consumo de sustancias en la esquizofrenia afecta a hombres y a mujeres de forma similar (debut más precoz, mayor gravedad clínica, peor pronóstico, etc. (Mueser et al., 1990)). No se encontraron diferencias de género en el número de días de consumo ni en la gravedad del mismo evaluado mediante el ASI (Gearon y Bellack, 2000). Aunque la evolución clínica es similar, existe mayor proporción de mujeres casadas $(48,5 \%$ vs $21,4 \%)$; ( $58,8 \%$ vs $41,2 \% p=0,006)$, con mayor número de hijos y a su cargo (Brunette y Drake, 1998; Brunette y Drake, 1997). El consumo de cannabis se ha relacionado con el debut precoz de la esquizofrenia en ambos sexos. En las mujeres, el uso diario de cannabis ( $z=-$ $1,92 ; p=0,054)$, se asoció a un mayor riesgo relativo de inicio de psicosis (hazard ratio $=5,15$ ) que en los hombres (hazard ratio $=3,36$ ) sin encontrar diferencias para el resto de formas de consumos de cannabis (Compton, Kelley, Ramsay, Pringle, Goulding, Esterberg et al., 2009). Las mujeres parecen ser más vulnerables a presentar sintomatología psicótica más rápidamente que los hombres (Gearon y Bellack, 2000). El género mujer deja de ser un factor de buen pronóstico cuando la esquizofrenia se acompaña de un TUS (Gearon y Bellack, 2000) debido al mayor número de hospitalizaciones, de síntomas positivos y peor nivel de funcionamiento global (Gearon y Bellack, 2000). El número medio de crímenes cometidos fue superior en los hombres psicóticos duales $(13,9$ vs 42,$6 ; p=0,01)$ (Brunette y Drake, 1998). Mientras que las mujeres sufrieron más agresiones (32,4\% vs $16,8 \%$; $p=0,04)$ y presentaron más historia de abuso físico y/o sexual (Brunette y Drake, 1998). Las mujeres psicóticas duales padecieron más problemas médicos (40,5\% vs 23,7\%; $d f=1 ; p=0,04)$ (Brunette y Drake, 1997).

\section{Trastornos afectivos}

Los trastornos afectivos en consumidores de sustancias son muy prevalentes. Alrededor del 30-50\% presentan un trastorno depresivo (Regier et al., 1990). Los trastornos afectivos duales siguen siendo, al igual que en la población general, más frecuentes en la mujer, excepto el trastorno bipolar tipo I (Cornelius, Jarrett, Thase, Fabrega, Haas, Jones-Barlock et al., 1995; Kessler, Crum, Warner, Nelson, Schulenberg, y Anthony, 1997).

La mayoría de estudios independientemente del tipo de muestra analizada coinciden en que los trastornos afectivos son más frecuentes en la mujer que en el varón (Tabla 2). Las mujeres alcohólicas en relación a los hombres presentaron una forma de beber más compulsiva, con ansiedad premórbida y depresión primaria (Dunne, Galatopoulos, y Schipperheijn, 1993). Además, el riesgo y las tasas de suicidio fueron superiores en las mujeres duales con clínica depresiva (Rounsaville, 2007) siendo el riesgo en éstas de 4,4 (1,9-10,2); $p=0,001$ (Chiang et al., 2007). 
Tabla 2. Distribución de los trastornos afectivos duales según género.

\begin{tabular}{|c|c|c|c|c|}
\hline Autor & Año & Muestra & Instrumentos & Resultados \\
\hline Chiang et al. & 2007 & $\begin{array}{l}155(123 \mathrm{H}, 32 \mathrm{M}) \text {. } \\
\text { Abuso/Depend. heroina } \\
\text { Tratamiento ambulatorio }\end{array}$ & MINI & $4,7(1,9-11,6) ; p=0,001$ (excluyendo el trastorno bipolar) \\
\hline Conner et al. & 2009 & $\begin{array}{l}\text { Se analizan } 60 \text { estudios. } \\
\text { Pacientes consumidores de } \\
\text { cocaína }\end{array}$ & $\begin{array}{l}\text { BDI, SCID, PRISM, BSI, } \\
\text { HRSD, HSCLE }\end{array}$ & $\begin{array}{l}\text { Se observó mayor grado de clínica depresiva en las mujeres } \\
\text { cocainómanas }(K=13, r=0,14(0,11-0,16), Z=10.04 ; p<0.001) \text {. }\end{array}$ \\
\hline Cornelius et al. & 1995 & $\begin{array}{l}604 \text { alcohólicos tratamiento } \\
\text { hospitalario psiquiátrico }\end{array}$ & Criterios DSM-IV & $\begin{array}{l}\text { Las mujeres alcohólicas presentaron más depresión que los } \\
\text { hombres }(F=4.28, d f=1,596, p=0,039) .\end{array}$ \\
\hline Kessler et al. & 1997 & $\begin{array}{l}8098 \text { entrevistados } \\
\text { Población general }\end{array}$ & CIDI & $\begin{array}{c}\text { El } 34,5 \% \text { mujeres con abuso de alcohol vs. } 10,2 \% \text { de hombres } \\
\text { ( } p<0,05) \text { y el } 53,5 \% \text { mujeres con dependencia al alcohol vs. } 28,1 \% \text { de } \\
\text { hombres }(p<0,05) \text { presentó clínica depresiva. }\end{array}$ \\
\hline Schutte et al. & 1997 & $\begin{array}{l}219 \mathrm{M}, 220 \mathrm{H} \\
\text { tratamiento ambulatorio TUS }\end{array}$ & & $\begin{array}{l}\text { Las mujeres refirieron más clínica depresiva a nivel basal que los } \\
\text { hombres (Media }=17,78 \pm 6,36 \text { vs. } 14,32 \pm 7,23 ; p<0,05 \text { ) }\end{array}$ \\
\hline Tomasson y Vaglum & 1995 & $\begin{array}{l}351 \text { ingresados U. DTX } \\
\text { (71\% hombres) }\end{array}$ & $\begin{array}{l}\text { MMSE } \\
\text { DSM-III-R }\end{array}$ & $26,9 \%$ hombres vs. $49 \%$ mujeres; $p<0,0001$. \\
\hline
\end{tabular}

H:hombres; M:mujeres: U. DTX: Unidad de desintoxicación.

Tabla. 3 Características de las mujeres bipolares duales

\begin{tabular}{|c|c|c|}
\hline \multirow{5}{*}{ Antecedentes } & Historia de abuso físico o sexual & \multirow{10}{*}{ MAYOR COMPLEJIDAD DE MANEJO } \\
\hline & Policonsumo & \\
\hline & Historia familiar de alcoholismo & \\
\hline & Episodios depresivos previos & \\
\hline & Fobia social & \\
\hline \multirow{5}{*}{ Clínica } & Mayor sintomatología mixta & \\
\hline & Mayor riesgo de ciclación rápida & \\
\hline & Más intentos autoliticos & \\
\hline & Menos actos violentos & \\
\hline & Menos problemas legales & \\
\hline
\end{tabular}

Se ha propuesto que el TUS de las mujeres con un trastorno depresivo dual es secundario al trastorno mental y que aparecería como una posible forma de automedicación, con el objetivo de aliviar el estrés emocional. Hanna y col. (1997) realizaron el análisis de las diferencias de género entre tres grupos de pacientes: el primero con depresión primaria; el segundo con TUS y depresión concurrente; y el tercero con depresión secundaria. Observaron que la proporción de mujeres en el grupo primario fue superior (54\% mujeres v $46 \%$ hombres) comparado con el grupo de depresión secundaria (57\% hombres v $43 \%$ mujeres) siendo las diferencias estadisticamente significativas (Hanna y Grant, 1997). En el varón al ser la depresión secundaria al consumo se la considera más refractaria (Brady et al., 1993).

Se ha establecido una relación entre la sintomatología ansiosa concurrente con el trastorno depresivo y el consumo de alcohol según el género. En los hombres se establece una relación lineal (a más ansiedad más consumo de alcohol) que no se cumple en las mujeres dado que en ellas el consumo desciende cuando el nivel de ansiedad es muy elevado (Fischer y Goethe, 1998).

Según los expertos encuestados en un estudio Español más del 20\% de los pacientes bipolares consumian sustancias (Casas, Franco, Goikolea, Jimenez-Arriero, 
Martinez-Raga, Roncero, et al. 2008). El trastorno bipolar dual es más frecuente en hombres que en mujeres (56\% vs 43\%) (Frye, Altshuler, McElroy, Suppes, Keck, Denicoff et al., 2003), a pesar de ser la prevalencia del trastorno Bipolar tipo I en no consumidores igual entre géneros (Baldassano, Marangell, Gyulai, Ghaemi, Joffe, Kim et al., 2005). El 29,1\% de las mujeres bipolares presentaron historia de consumo de alcohol frente al $49 \%$ de los hombres $(p=0,001)$. Por otra parte, comparado con la población general las mujeres presentaron 7,35 veces más riesgo de presentar alcoholismo y los hombres de 2,77 siendo las diferencias significativas (Frye et al., 2003).

El manejo clínico de las pacientes bipolares duales es más complejo dada la peor evolución clínica (Casas et al., 2008) (Tabla 3). Las mujeres presentaron más episodios depresivos que los hombres ( $93 \%$ vs $54 \% ; p=0,001)$ y el número medio de sintomas depresivos fue significativamente superior en éstas $((16,76 \pm 6,43)$ vs. $(11,88 \pm 7,48) ; t=2,79 ; d f=1,63$; $p=0,007$ ) (Salloum, Cornelius, Mezzich, Kirisci, Daley, Spotts et al., 2001).

\section{Trastornos de ansiedad}

Los trastornos de ansiedad están muy asociados al consumo de sustancias legales, ilegales y también al abuso de fármacos analgésicos por parte de las mujeres (Tucker y Westermeyer, 1995). Diversos estudios coinciden en que la ansiedad dual es más frecuente en mujeres ( $53 \%$ de mujeres vs. $47 \%, p<0,05)$ y $(73,5 \%$ mujeres vs $62,6 \%$ hombres, $\mathrm{p}<0,05)$ ) (Tomasson y Vaglum, 1995; Tucker y Westermeyer, 1995). Sobretodo se encontraron diferencias de género en la presencia de clínica ansiosa cuando la sustancia de estudio fue el alcohol (48,8\% mujeres con abuso vs el $22,7 \%$ de hombres y $60,7 \%$ de mujeres con dependencia vs. $35,8 \%$ de hombres (Kessler et al., 1997). Además, la proporción de mujeres duales (53\%) fue mayor que la proporción de mujeres en el grupo no dual (35\%) (Westermeyer, Tucker, y Nugent, 1995). Al igual que ocurre con el trastorno depresivo, las mujeres suelen debutar primero con el trastorno de ansiedad (Hesselbrock, Meyer, y Keener, 1985).

Aunque existen pocos estudios en pacientes con trastorno obsesivo compulsivo (TOC), se han detectado diferencias de género en algún subtipo. Las mujeres acumuladoras presentaron mayores tasas de trastorno por uso de sustancias $(F=12,03, p<, 001)$ que las no acumuladoras y abuso de alcohol $(F=4,64, p<0,03)$ (Wheaton, Timpano, Lasalle-Ricci, y Murphy, 2008).

La presencia de antecedentes traumáticos en los pacientes con patología psiquiátrica es elevada y predomina en las mujeres. El número de mujeres que presenta un antecedente traumático es similar entre las duales y no duales. Sin embargo, las primeras presentan mayor número de traumas que las que sólo presentan un TUS (Reynolds, Mezey, Chapman, Wheeler, Drummond, y Baldacchino, 2005). Gearon y cols (2003a) observaron que en una muestra de 54 pacientes diagnosticadas de esquizofrenia y trastorno esquizoafectivo, mayoritariamente dependientes de cocaína, el 96\% presentaron un antecedente traumático, aunque sólo en el $46 \%$ de la muestra total se le diagnosticó el TEPT, siendo en las mujeres dos veces más prevalente. Entre los traumas causantes de TPET, las mujeres presentaron más antecedentes de abuso sexual y/o físico y los hombres de crimenes o desastres naturales (Gearon, Kaltman, Brown, y Bellack, 2003a). El ser mujer aumenta la probabilidad de desarrollar un TEPT (beta $=2,106 ; p<0,05$ ) (Montoya, Covarrubias, Patek, y Graves, 2003). En las mujeres drogodependientes que aparece un TPET la discapacidad a nivel social, laboral y familiar es mayor (Gearon et al., 2003a).

La prevalencia del trastorno de ansiedad generalizada (TAG) en mujeres es superior que en hombres (5,3\% vs $2,8 \%$ respectivamente). Sin embargo, la presencia de TAG en los hombres aumenta el riesgo de comorbilidad con el trastorno por consumo de alcohol y otras drogas $(O R=2,7(1,7-4,1))$. Las mujeres con TAG presentaron significativamente mayor comorbilidad con otros trastornos afectivos (excepto el trastorno bipolar) y otros trastornos de ansiedad a excepción de la fobia social. Además, las mujeres duales presentaron mayor número de quejas somáticas siendo el grado de afectación mayor para las mujeres con TAG que para los hombres (Vesga-Lopez, Schneier, Wang, Heimberg, Liu, Hasin et al., 2008).

\section{CONCLUSIONES}

El estudio de las diferencias epidemiológicas, diagnósticas y clínicas según el género, en los pacientes duales, se puede realizar desde dos perspectivas. En primer lugar, mediante la evaluación de las diferencias entre hombres y mujeres duales según el trastorno mental; y en segundo lugar, comparando, en función del género, los pacientes duales de los no duales. Ambas perspectivas de estudio son complejas. Existen escasos trabajos sistemáticos, las muestras son en la mayoría de casos reducidas y heterogéneas, ello explica la dificultad en establecer conclusiones.

Existe controversia acerca de si la patología dual es más frecuente en varones o en mujeres, existiendo diferencias en función del lugar donde los estudios se realicen; ya sea en unidades de psiquiatría general, donde hay más hombres duales que mujeres, o en unidades de drogodependencias donde se detecta lo contrario. Los datos epidemiológicos generales, según diagnóstico, no presentan grandes variaciones entre pacientes duales y no duales. Los hombres duales presentan mayor prevalencia de esquizofrenia y las mujeres de trastornos afectivos y de ansiedad como sucede en la población general. Sociodemográficamente no existen grandes diferencias entre hombres y mujeres duales, excepto que las mujeres duales tienen más hijos a su cargo, mayor riesgo de victimización que los hombres duales y menores problemas legales. Todos los estudios coinciden en que los pacientes varones duales presentan mayor patrón de policonsumo. Por otra parte, no se pueden establecer conclusiones acerca de las diferencias de uso de 
las sustancias consumidas según género en los pacientes duales dada la variabilidad de las muestras.

Las mujeres psicóticas duales tienen un pronóstico similar al de los hombres duales y claramente peor que el de las psicóticas sin TUS. Además, tienen una mayor carga familiar, más historia de agresiones y problemas médicos.

En relación a los pacientes con un trastorno afectivo que consumen, se conoce que las mujeres presentan un consumo relacionado con la ansiedad. Tienen mayor riesgo de realizar intentos suicidas o suicidios consumados y siguen más frecuentemente un patrón de automedicación y consumo compulsivo. Se podría hipotetizar que existe un origen etiopatogénico diferente del TUS según el género, ya que la mayoria de estudios observan que los trastornos afectivos en las mujeres son primarios, a diferencia de los hombres, en los que son predominante secundarios al consumo y más difíciles de tratar. Desde la perspectiva psicopatológica, el riesgo de presentar un TUS, cambia en función del género. Por ejemplo, las mujeres bipolares tienen mayor riesgo de presentar un TUS comparado con las de la población general, en relación a los hombres.

Cuando se estudian los trastornos de ansiedad se conoce que las mujeres duales presentan una prevalencia similar de TEPT, que las que sólo tienen una patología mental, pero superior que las que tienen un TUS. Otros trastornos como el TAG, de predomino habitualmente femenino, son más prevalentes en hombres duales.

Por todo lo anteriormente descrito, se debe profundizar en el estudio de las diferencias de género en patología dual, ya que se observan variaciones, que pueden influir en el diagnóstico, el tratamiento y en la planificación de los servicios.

\section{AGRADECIMIENTOS Y CONFLICTOS DE INTERES}

Se agradece a la Dra. Gideoni Fuste, psiquiatra del CAS de Vall d'Hebron, su colaboración en la revisión del manuscrito final.

Ninguno de los autores tiene conflictos de interés.

\section{REFERENCIAS}

Abram, K. M., Teplin, L. A., y McClelland, G. M. (2003). Comorbidity of severe psychiatric disorders and substance use disorders among women in jail. American Journal of Psychiatry, 160, 1007-1010.

Baldassano, C. F., Marangell, L. B., Gyulai, L., Ghaemi, S. N., Joffe, H., Kim, D. R.,... Cohen, L. S. (2005). Gender differences in bipolar disorder: Retrospective data from the first 500 STEP-BD participants. Bipolar Disorders, 7, 465-470.

Brady, K. T., Grice, D. E., Dustan, L., y Randall, C. (1993). Gender differences in substance use disorders. American Journal of Psychiatry, 150, 1707-1711.

Brunette, M. F., y Drake, R. E. (1997). Gender differences in patients with schizophrenia and substance abuse. Comprehensive Psychiatry, 38, 109-116.
Brunette, M., y Drake, R. E. (1998). Gender differences in homeless persons with schizophrenia and substance abuse. Community Mental Health Journal, 34, 627-642.

Cantwell, R., Brewin, J., Glazebrook, C., Dalkin, T., Fox, R., Medley, I., y Harrison, G. (1999). Prevalence of substance misuse in firstepisode psychosis. British Journal of Psychiatry, 174, 150-153.

Casas, M., Franco, M. D., Goikolea, J. M., Jimenez-Arriero, M. A., Martinez-Raga, J., Roncero, C., Szerman, N. (2008). Bipolar disorder associated to substance use disorders (dual diagnosis). Systematic review of the scientific evidence and expert consensus. Actas Españolas de Psiquiatría, 36, 350-361.

Chiang, S. C., Chan, H. Y., Chang, Y. Y., Sun, H. J., Chen, W. J., y Chen, C. K. (2007). Psychiatric comorbidity and gender difference among treatment-seeking heroin abusers in taiwan. Psychiatry and Clinical Neurosciences, 61, 105-111.

Claassen, C. A., Gilfillan, S., Orsulak, P., Carmody, T. J., Battaglia, J., y Rush, A. J. (1997). Substance use among patients with a psychotic disorder in a psychiatric emergency room. Psychiatric Services, 48, 353-358.

Compton, M. T., Kelley, M. E., Ramsay, C. E., Pringle, M., Goulding, S. M., Esterberg, M. L.,...Walker, E. F. (2009). Association of preonset cannabis, alcohol, and tobacco use with age at onset of prodrome and age at onset of psychosis in first-episode patients. American Journal of Psychiatry, 166, 1251-1257.

Comtois, K. A., y Ries, R. K. (1995). Sex differences in dually diagnosed severely mentally ill clients in dual-diagnosis outpatient treatment. American Journal of Addiction, 4, 245-253.

Conner, K. R., Pinquart, M., y Gamble, S. A. Meta-analysis of depression and substance use among individuals with alcohol use disorders. (2009). Journal of Substance Abuse Treatment, 37, 127-137.

Cornelius, J. R., Jarrett, P. J., Thase, M. E., Fabrega, H. Jr., Haas, G. L., Jones-Barlock, A.,...Ulrich, R. F. (1995). Gender effects on the clinical presentation of alcoholics at a psychiatric hospital. Comprehensive Psychiatry, 36, 435-440.

Denier, C. A., Thevos, A. K., Latham, P. K., y Randall, C. L. (1991). Psychosocial and psychopathology differences in hospitalized male and female cocaine abusers: A retrospective chart review. Addictive Behaviors, 16, 489-496.

DiNitto, D. M., Webb, D. K., y Rubin, A. (2002). Gender differences in dually-diagnosed clients receiving chemical dependency treatment. Journal of Psychoactive Drugs, 34, 105-117.

Dunne, F. J., Galatopoulos, C., y Schipperheijn, J. M. (1993). Gender differences in psychiatric morbidity among alcohol misusers. Comprehensive Psychiatry, 34, 95-101.

Fischer, E. H., y Goethe, J. W. (1998). Anxiety and alcohol abuse in patients in treatment for depression. American Journal of Drug and Alcohol Abuse, 24, 453-463.

Frye, M. A., Altshuler, L. L., McElroy, S. L., Suppes, T., Keck, P. E., Denicoff, K.,...Post, R. M. (2003). Gender differences in prevalence, risk, and clinical correlates of alcoholism comorbidity in bipolar disorder. American Journal of Psychiatry, 160, 883-889.

Gearon, J. S., y Bellack, A. S. (2000). Sex differences in illness presentation, course, and level of functioning in substanceabusing schizophrenia patients. Schizophrenia Research, 43, 65-70.

Gearon, J. S., Kaltman, S. I., Brown, C., y Bellack, A. S. (2003a). Traumatic life events and PTSD among women with substance use disorders and schizophrenia. Psychiatric Services, 54, 523-528. 
Gearon, J. S., Nidecker, M., Bellack, A., y Bennett, M. (2003b). Gender differences in drug use behavior in people with serious mental illnesses. American Journal on Addictions, 12, 229-241.

Gual, A. (2007). Dual diagnosis in Spain. Drug and Alcohol Review, 26, $65-71$.

Hanna, E. Z., y Grant, B. F. (1997). Gender differences in DSM-IV alcohol use disorders and major depression as distributed in the general population: Clinical implications. Comprehensive Psychiatry, 38, 202-212.

Hesselbrock, M. N., Meyer, R. E., y Keener, J. J. (1985). Psychopathology in hospitalized alcoholics. Archives of General Psychiatry, 42, 1050-1055.

Kessler, R. C., Crum, R. M., Warner, L. A., Nelson, C. B., Schulenberg, J., y Anthony, J. C. (1997). Lifetime co-occurrence of DSM-III-R alcohol abuse and dependence with other psychiatric disorders in the national comorbidity survey. Archives of General Psychiatry, 54, 313-321.

Krakow, D. S., Galanter, M., Dermatis, H., y Westreich, L. M. (1998). HIV risk factors in dually diagnosed patients. American Journal of addiction, 7, 74-80.

Landheim, A. S., Bakken, y Vaglum, P. (2003). Gender differences in the prevalence of symptom disorders and personality disorders among poly-substance abusers and pure alcoholics. Substance abusers treated in two countries in Norway. European Addiction Research, 9, 8-17.

Montoya, I. D., Covarrubias, L. D., Patek, J. A., y Graves, J. A. (2003). Posttraumatic stress disorder among hispanic and africanamerican drug users. American Journal of Drug and Alcohol Abuse, 29, 729-741.

Mowbray, C. T., Ribisl, K. M., Solomon, M., Luke, D. A., y Kewson, T. P. (1997). Characteristics of dual diagnosis patients admitted to an urban, public psychiatric hospital: An examination of individual, social, and community domains. American Journal of Drug and Alcohol Abuse, 23, 309-326.

Mueser, K. T., Yarnold, P. R., y Bellack, A. S. (1992). Diagnostic and demographic correlates of substance-abuse in schizophrenia and major affective-disorder. Acta Psychiatrica Scandinavica, 85, 48-55.

Mueser, K. T., Yarnold, P. R., Levinson, D. F., Singh, H., Bellack, A. S., Kee, K.,...Yadalam, K. G. (1990). Prevalence of substance abuse in schizophrenia: Demographic and clinical correlates. Schizophrenia Bulletin, 16, 31-56.

Regier, D. A., Farmer, M. E., Rae, D. S., Locke, B. Z., Keith, S. J., Judd, L. L., y Goodwin, F. K. (1990). Comorbidity of mental disorders with alcohol and other drug abuse. results from the epidemiologic catchment area (ECA) study. Journal of the American Medical Association, 264, 2511-2518.

Reynolds, M., Mezey, G., Chapman, M., Wheeler, M., Drummond, C., y Baldacchino, A. (2005). Co-morbid post-traumatic stress disorder in a substance misusing clinical population. Drug and Alcohol Dependence, 77, 251-258.
Rodriguez-Jimenez, R., Aragues, M., Jimenez-Arriero, M. A., Ponce, G., Munoz, A., Bagney, A.,...Palomo, T. (2008). Dual diagnosis in psychiatric inpatients: Prevalence and general characteristics [Patologia dual en pacientes psiquiatricos hospitalizados: prevalencia y caracteristicas generales]. Investigacion Clinica, 49, $195-205$.

Rounsaville, B. J. (2007). DSM-V research agenda: Substance abuse/ psychosis comorbidity. Schizophrenia Bulletin, 33, 947-952.

Salloum, I. M., Cornelius, J. R., Mezzich, J. E., Kirisci, L., Daley, D. C., Spotts, C. R., ..., Zuckoff, A. (2001). Characterizing female bipolar alcoholic patients presenting for initial evaluation. Addictive Behaviors, 26, 341-348.

Sanabria, M. A., Díaz, O., Varela, J., y Brana, T. (2002). Relevancia para el tratamiento de la sintomatología depresiva de los pacientes adictos. Adicciones, 14, 25-31.

Seedat, S., Scott, K. M., Angermeyer, M. C., Berglund, P., Bromet, E. J., Brugha, T. S.,...Kessler, R. C. (2009). Cross-national associations between gender and mental disorders in the world health organization world mental health surveys. Archives of General Psychiatry, 66, 785-795.

Schutte, K. K, Hearst, J., y Moss, R. H. (1997). Gender differences in the relations between depressive symptoms and drinking behavior among problem drinkers: a three-wave study. Journal of Consulting and Clinical Psychology, 65, 392-404.

Tomasson, K., y Vaglum, P. (1995). A nationwide representative sample of treatment-seeking alcoholics - a study of psychiatric comorbidity. Acta Psychiatrica Scandinavica, 92, 378-385.

Tucker, P., y Westermeyer, J. (1995). Substance abuse in patients with comorbid anxiety disorder. American Journal of Addiction, 4 , 226-233.

Vesga-Lopez, O., Schneier, F. R., Wang, S., Heimberg, R. G., Liu, S. M., Hasin, D. S., y Blanco, C. (2008). Gender differences in generalized anxiety disorder: Results from the national epidemiologic survey on alcohol and related conditions (NESARC). Journal of Clinical Psychiatry, 69, 1606-1616.

Westermeyer, J., Tucker, P., y Nugent, S. (1995). Comorbid anxiety disorder among patients with substance abuse disorders. risk factors on initial evaluation. American Journal of Addiction, 4, 97-106.

Westreich, L., Guedj, P., Galanter, M., y Baird, D. (1997). Differences between men and women in dual-diagnosis treatment. American Journal on Addictions, 6, 311-317.

Wheaton, M., Timpano, K. R., Lasalle-Ricci, V. H., y Murphy, D. (2008). Characterizing the hoarding phenotype in individuals with OCD: Associations with comorbidity, severity and gender. Journal of Anxiety Disorders, 22, 243-252.

Zlotnick, C., Clarke, J. G., Friedmann, P. D., Roberts, M. B., Sacks, S., y Melnick, G. (2008). Gender differences in comorbid disorders among offenders in prison substance abuse treatment programs. Behavioral Science and the Law, 26, 403-412. 